\title{
Influence of Gas Pressure on Coulometric Trace Humidity Measurement
}

\author{
M. Detjens, C. Tiebe, U. Banach \\ Federal Institute for Materials Research and Testing, Berlin, Germany, \\ marc.detjens@bam.de
}

\begin{abstract}
:
Planar coulometric sensors were investigated in humidified synthetic air at various absolute gas pressures, i. e. 2 bar, 5, bar, 10 bar, and 15 bar. Humidified gas flow at adjusted gas pressure was split into two flows, one passed a coulometric sensor and the other one passed a reference hygrometer after decompression. Both signals were recorded and then compared after calculation of resulting frost point temperature. Calculation is based on a calibration function obtained at ambient pressure. Comparison showed that an increasing pressure resulted in a higher derivation between sensor signal (calculated frost point temperature) and reference frost point temperature. At an absolute pressure of 2 bar the differences were minor in consideration of the uncertainty, however at 15 bar the differences were $6.77 \mathrm{~K}$. Nevertheless, it was possible to measure the gas humidity at higher pressure with coulometric trace humidity sensors.
\end{abstract}

Key words: Coulometric sensors, Trace humidity measurement, Gas pressure influence.

\section{Introduction}

Measurement of trace humidity in compressed gases is a challenging task. Even small amounts of water vapour can negatively influence processes and its products. Trace humidity of gases covers frost point temperature $t_{f}$ less than $-13^{\circ} \mathrm{C}$, which is equivalent to a water vapour mole fraction $x_{v}$ and an absolute humidity $d_{v}$ below $2000 \mu \mathrm{mol} \cdot \mathrm{mol}^{-1}$ or $1.45 \mathrm{~g} \cdot \mathrm{m}^{-3}$, respectively [1]. Often, processes are performed at gas pressures that differ from ambient pressure ( $p=1.013$ bar) and it is required to measure humidity content directly in the gas flow at higher pressures. Thus, used humidity sensors should be unaffected by gas pressure or its influence should be known.

Coulometric sensors are suitable hygrometers for continuous measurement of humidity in the frost point temperature range from $-15^{\circ} \mathrm{C}$ to $-90{ }^{\circ} \mathrm{C}$ under rough process conditions. The setup consists of a sensor element, voltage supply, digital multimeter, and a regulated gas flow over the sensor. Nevertheless, coulometric sensors need to be calibrated for an accurate measurement of humidity. Sensor signal is influenced by temperature, voltage supply, sensor coating, gas flow and by the gas pressure. Useful metrological range of these hygrometers is specified by standard VDI/VDE
3514-2 [2]. It states an operation range for the gas pressure from 0,001 bar to 10 bar.

Purpose of this study is to investigate the gas pressures influence on the sensor signal in comparison to ambient pressure. Furthermore, the attempt to enlarge the standard to pressures up to 15 bar.

\section{Coulometric Sensors}

Coulometric sensors were described by Keidel as a method to determine trace humidity in 1959 [3-5]. It is based on the electrolysis of absorbed water which is decomposed to hydrogen and oxygen at a voltage of at least $2 \mathrm{~V}$ (DC). Process can be described by means of Faraday's law that states a correlation between the amount of electrical charge $Q$ and water substance $n$ water as shown in eq. (1).

$Q=n$ water $\cdot F \cdot z(1)$

In equilibrium state, the electrical current $I$ is proportional to the water mass flow $\dot{m}$ water.

\section{Humidity Generation}

The humidity generation facility at higher pressures consists of mass flow controllers, digital manometer, control valves, reference hygrometer. Generation is controlled with a Digital Single-Channel Control Module (E7000, Bronkhorst), which includes two mass flow controllers (F-231M, Bronkhorst), digital 
electronic pressure meter (EL-PRESS P-532C, Bronkhorst) and two control valves (F-033, Bronkhorst). Generated humidity is divided into two flows; one passes a coulometric sensor at adjusted gas pressure and the other flow passes a reference hygrometer (DP30, MBW) that measures the frost point temperature after gas decompression. Gas pressure in the system is measured by a digital manometer (Lex 1, Keller).

\section{Results}

For the experiments, the sensor was exposed to various humidity levels and to the absolute gas pressures of 2 bar, 5 bar, 10 bar, and 15 bar. Fig. 1 . shows as example a measurement of humidity at an absolute pressure of 5 bar and 15 bar. Reference measurement of humidity is shown by the yellow line and the red line shows calculated signal of the coulometric sensor. Basis for the calculated frost point temperature is a non-linear calibration function, see eq. (2), obtained from previous measurements at ambient pressure (1.013 bar) with an expanded uncertainty $U\left(T_{f}\right)$ of $1.3 \mathrm{~K}$.

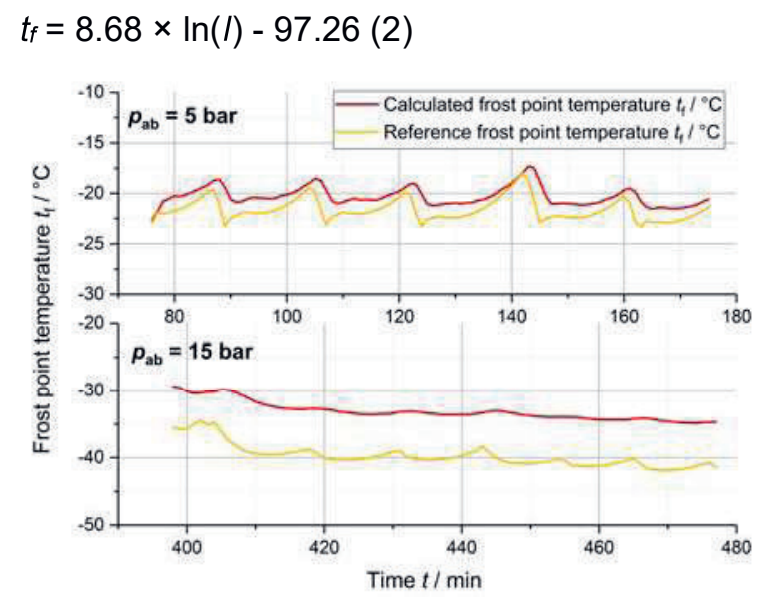

Fig. 1. Measurement of the humidity, represented as frost point temperature $t_{f}$, at absolute pressure of 5 bar and 15 bar.

Results show that difference between reference humidity value and calculated humidity level increase with higher gas pressures. At an absolute pressure of 2 bar, the difference is approximately $1 \mathrm{~K}$ and thus below measurement uncertainty. At an absolute pressure of 5 bar, the difference is approximately $-2 \mathrm{~K}$. In contrast to that the difference is $-6.77 \mathrm{~K}$ at an absolute pressure of 15 bar, i. e. sensor measured higher frost point temperatures. Reason is the change of water vapour pressure due to total pressure change that results in a shift of the equilibrium state.

\section{Conclusion}

Results of the experiments show that it is possible to measure with this kind of coulometric sensors at higher pressures. Pressures above 10 bar were also possible. However, the excess pressures influence the measurement results. Coulometric sensors measure higher electric currents due to the change in water vapour pressure with increasing pressure. Additional measurements are necessary to fully understand and validate the influence on the measurement results.

\section{Acknowledgement}

The authors thank Thomas Bernstein, Sylvia Filarski and Jörg Schlischka for technical assistance. This work has been financially supported by the Federal Ministry for Economic Affairs and Energy (BMWi) - ZIM-project No. $2201085 K M 4$.

In memoriam of Dr. Thomas Hübert.

\section{References}

[1] VDI/VDE 3514-1:2016-11, Measurement of gas humidity - Characteristics and symbols, 2016.

[2] VDI/VDE 3514-1:2013-03, Measurement of gas humidity - Methods of measurement, 2013.

[3] F. A. Keidel, "Determination of water by direct amperometric measurement," Analytical Chemistry, vol. 31, no. 12, pp. 2043-2048, 1959.

[4] R. Wernecke and J. Wernecke, Industrial Moisture and Humidity Measurement: A Practical Guide. Wiley, 2014.

[5] J. Mitchell and D. M. Smith, Aquametry: a treatise on methods for the determination of water. Wiley, 1984. 